\title{
A novel approach to the repair of urethrocutaneous fistulae arising after abdominoperineal anorectal resection
}

\author{
Alaya Yassein, MD; Shawn Dason, MD; ${ }^{*}$ Stephen Kelly, MD, FRCSC; $;$ Timothy Davies, MD, FRCSC* \\ *Division of Urology, McMaster University, Hamilton, ON; *Division of Surgery, McMaster University, Hamilton, ON
}

Cite as: Can Urol Assoc J 2015;9(11-12):E879-81. http://dx.doi.org/10.5489/cuaj.2938 Published online December 14, 2015

\section{Abstract}

This case report describes a novel approach to the repair of perineal urethrocutaneous fistulae (UCF) after abdominoperineal resection (APR). A 62-year-old patient developed a UCF after an APR for rectal cancer complicated by perineal abscess formation. The patient presented with continuous urinary drainage from the fistula that persisted despite a number of conservative and surgical measures. The patient underwent successful repair of the urethrocutaneous fistula in prone position - an approach that has not previously been described in the literature. Repair was performed by the multidisciplinary team of a reconstructive urologist, colorectal surgeon, and plastic surgeon. Post-operative retrograde urethrogram demonstrated the absence of a persistent fistula tract and the patient has been continent for 18 months. The prone approach for UCF repair allows for excellent access to the fistula tract for posterior urethroplasty in a patient that has had prior APR.

\section{Introduction}

Urinary fistulae are an uncommon complication after rectal surgery. ${ }^{1}$ Fistulae may arise directly from injury to urinary tract structures or indirectly as a consequence of abscess formation. ${ }^{2}$ With increasing survivorship after rectal surgery, management of long-term urinary complications is becoming increasingly relevant. In this case report, a new prone approach to a perineal urethrocutaneous fistula (UCF) arising after abdominoperineal resection (APR) is described.

\section{Case report}

A 62-year-old male patient presented with high-volume continuous incontinence. This arose four months after an APR for a localized rectal cancer close to the anal verge.
He developed a postoperative perineal wound infection complicated by abscess formation and subsequent incontinence after the abscess ruptured. A diagnosis of UCF was confirmed with retrograde urethrography.

Over the course of two years, the patient was managed with a variety of conservative measures, including antibiotics, wound care, hyperbaric oxygen therapy, and catheter drainage. An injection of Floseal (Baxter, US) into the tract and an abdominal approach to UCF repair were also unsuccessful.

Due to the significant impact on quality of life resulting from this patient's incontinence, he requested another attempt at operative repair. The patient was assessed at our tertiary centre by a multidisciplinary team, including a reconstructive urologist, colorectal surgeon, and plastic surgeon. Magnetic resonance imaging was incorporated in the operative planning and demonstrated a tract from the apex of the prostate and distal prostatic urethra extending presacrally and terminating in an area of fibrosis in the perineum. Cystoscopy and retrograde urethrography were also performed preoperatively (Figure 1).

For the operation, the patient was positioned prone with hips abducted and slightly flexed. The mature epithelialized fistula tract was easily identified and had a diameter of 1 $\mathrm{cm}$ (Figure 2A). The tract was opened from the perineum to the coccyx and bluntly debrided along its length. Evicel (Ethicon, U.S.) was injected through the presacral tract to try to facilitate ablation of the cavity.

Dissection was then carried down to the prostatic urethra, where the area of fistulization was identified and consistent with the preoperative MRI images. A cystoscopically placed guidewire aided in identifying the fistula tract close to the urethra (Figure 2B). The urethral defect was closed with three non-overlapping layers of interrupted PDS sutures (Ethicon, U.S.). The closure was inspected urethroscopically and found to be water-tight and tension-free. Urethral and suprapubic catheters were placed for maximal urinary diversion. 


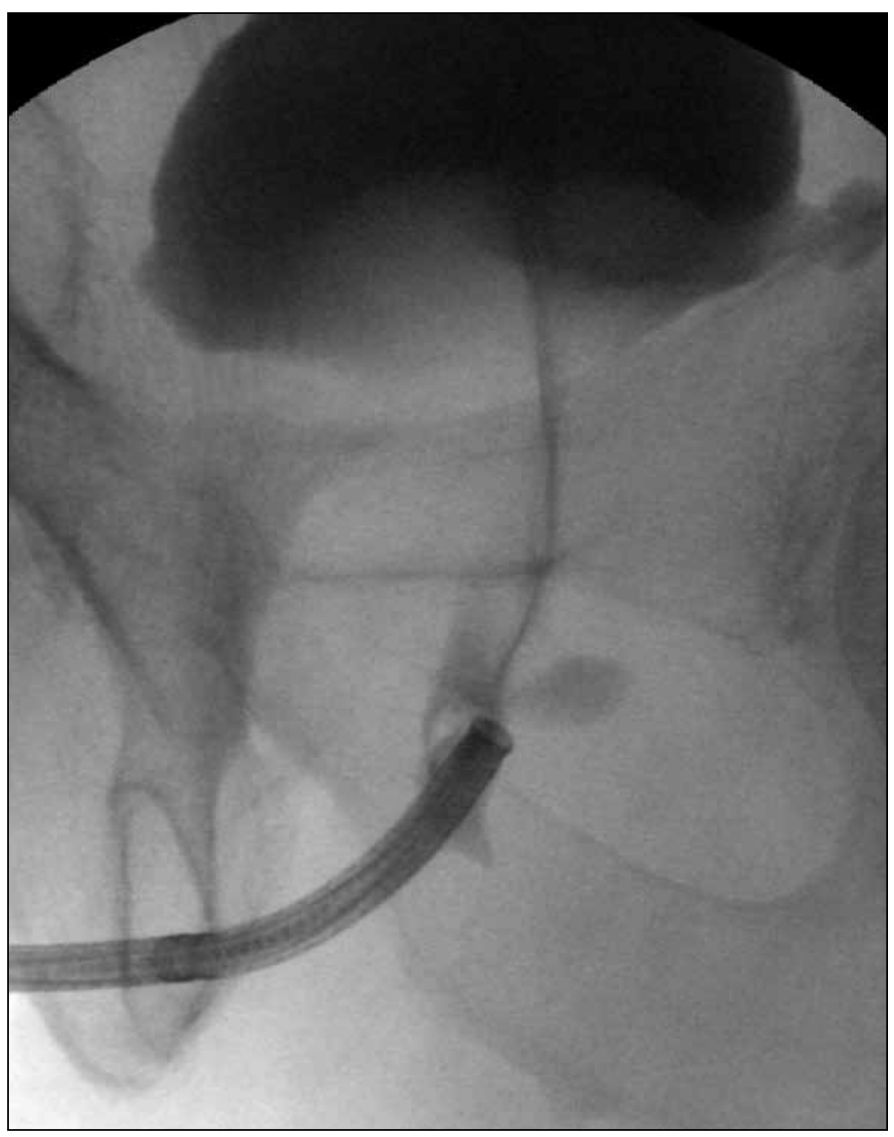

Fig. 1. A gentle preoperative retrograde urethrogram demonstrates the origin of the urethrocutaneous fistula in the prostatomembranous urethra.
To provide a barrier layer for the urethroplasty, bilateral pedicle flaps of perineal and ischiorectal fat were mobilized. One flap was sutured over the presacral fistula tract and the other covered the urethral closure (Figure 2C). The subcutaneous fat and skin were subsequently closed in two additional non-overlapping layers. The patient had difficulty weaning from ventilator postoperatively, but there were no other perioperative complications.

The patient went on to have contrast studies at six weeks postoperatively. With $200 \mathrm{cc}$ of contrast injected at supraphysiologic pressures, there was some small irregularity in the posterior urethra and some filling of a cavity next to the posterior urethra (Figure 3). Despite these early and nonphysiologic testing conditions, there was no visualized tract between the cavity and the posterior urethra and no leakage of contrast into the perineum.

After 18 months followup, the patient continues to be continent of urine and required no urinary pads. Voiding function was otherwise unchanged. The patient was satisfied with the overall outcome of this repair.

\section{Discussion}

Persistence of a perineal sinus after APR is thought to be related to chronic inflammation and fibrosis preventing wound healing. Risk factors for perineal sinuses include inadequate operative hemostasis, anatomical dead-space following resection, increased body mass index (BMI), preoperative radiotherapy, and diabetes. ${ }^{2,3}$ When a perineal sinus involves the urethra, a UCF results. Urethrocutaneous fistulae have also

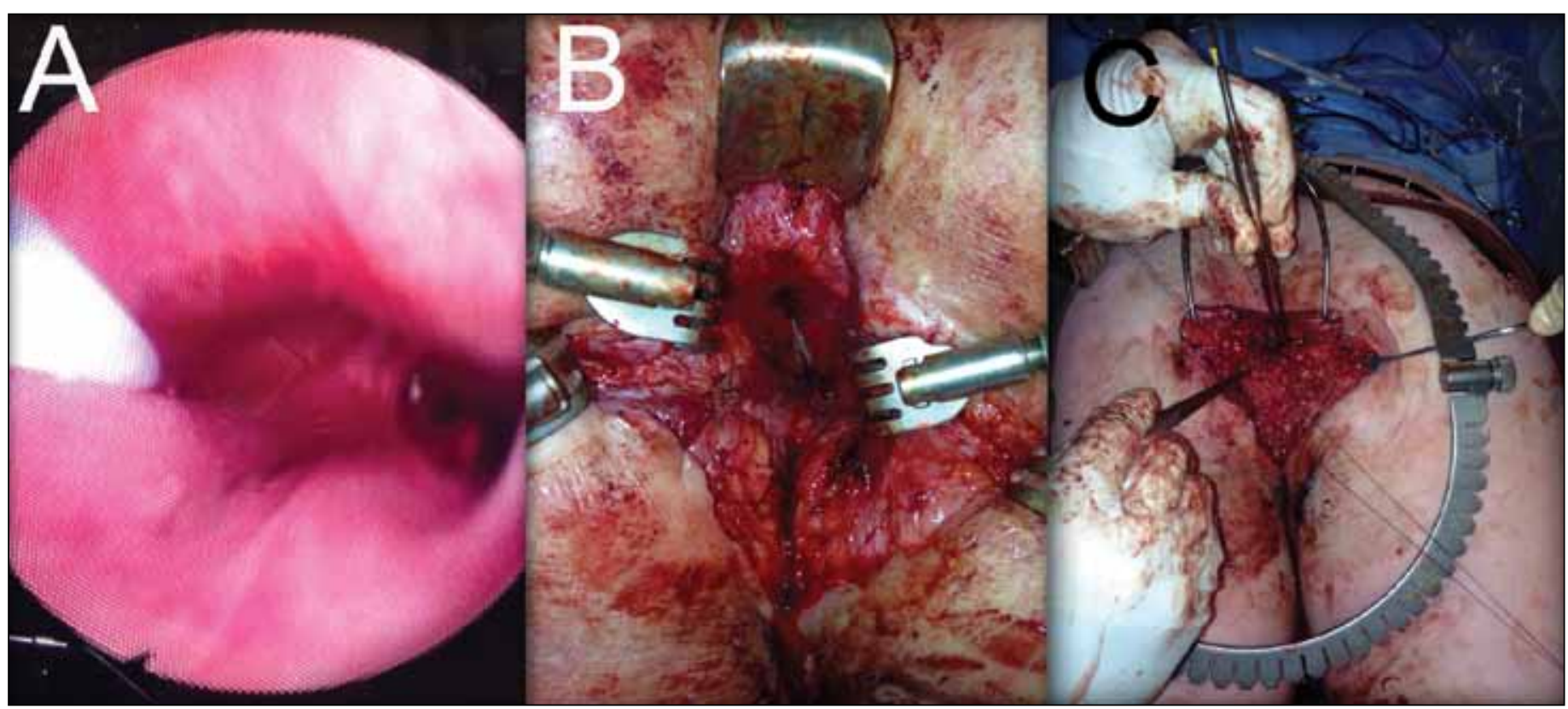

Fig. 2. A: Urethroscopic visualization of the mature fistula tract with probe in situ; $B$ : The patient is in prone positioning with the urethroscopically visualized probe in situ. The fistula is easily visualized superficially and at the centre of the operative field. C: The prone position allows access to an abundant local tissue flap for a tension-free fistula closure. 


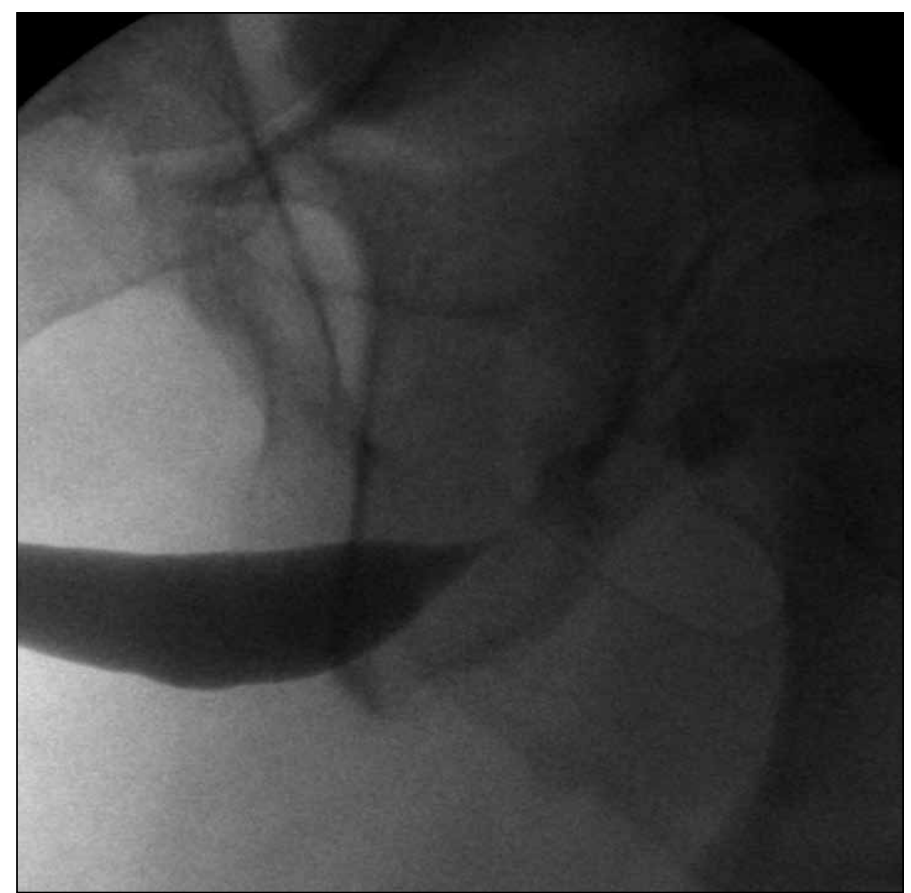

Fig. 3. Postoperative retrograde urethrogram injected using $200 \mathrm{cc}$ of dilute contrast at supraphysiologic pressures demonstrates persistence of a small cavity behind the posterior urethra, but no direct tract between the urethra and this cavity. The patient was free from perineal urinary leakage at this time and has been dry for 18 months.

been described as a "watering-can" perineum. ${ }^{4}$

Management of persistent perineal sinuses follows a stepwise approach from least to most invasive. Conservative measures include antibiotics, local wound care, vacuumassisted closure, ${ }^{2}$ fibrin glue,${ }^{5}$ and hyperbaric oxygen therapy. ${ }^{6}$ The additional principles of urinary diversion and management of distal obstruction are important when dealing with UCF.

The anatomy of UCF that develop after anorectal surgery is likely different than UCF arising from other etiologies, such as stricture disease or failed hypospadias repair. The latter will generally involve the anterior urethra and drain to the penis or scrotum. Previous descriptions of the operative repair of UCF have focused on these more common etiolo- gies of UCF. Urethrocutaneous fistulae arising after anorectal surgeries should involve the posterior urethra and exit near the perineal wound posterior to the perineal body.

The prone approach to repair of UCF has not previously been described. We decided upon this approach, as it was felt that the external opening of the tract was quite posterior, even for a patient placed in an extra-high dorsal lithotomy position. An abdominal approach had previously failed and was thought to be unsatisfactory for posterior urethroplasty. The lack of an anus and rectum facilitated access to the posterior urethra via this approach.

\section{Conclusion}

This report suggests that the prone approach to repair of UCF arising after APR is feasible. This approach provides excellent access to both the perineal fistula opening and the posterior urethra.

Competing interests: The authors declare no competing financial or personal interests.

This paper has been peer-reviewed.

\section{References}

1. Gillespie L, Barbaric Z, Raz S. Effect of abdominal perineal resection on genitourinary tract. Urology 1985;25:259-63. http://dx.doi.org/10.1016/0090-4295(85)90324-3

2. Lohsiriwat $V$. Persistent perineal sinus: incidence, pathogenesis, risk factors, and management. Surg Today 2009;39:189-93. http://dx.doi.org/10.1007/s00595-008-3846-z

3. Christian CK, Kwaan MR, Betensky RA, et al. Risk factors for perineal wound complications following abdominoperineal resection. Dis Colon Rectum 2005;48:43-8. http://dx.doi.org/10.1007/s10350004-0855-x

4. Pandhi D, Reddy B. Watering can perineum-a forgotten complication of gonorrhoea. J Eur Acad Dermatol Venereol 2002;16:486-7. http://dx.doi.org/10.1046/i.1468-3083.2002.00652.x

5. Zmora 0, Mizrahi N, Rotholtz N, et al. Fibrin glue sealing in the treatment of perineal fistulas. Dis Colon Rectum 2003;46:584-9. httrp://dx.doi.org/10.1007/s10350-004-6612-3

6. Capelli-Schellpfeffer M, Gerber GS. The use of hyperbaric oxygen in urology. J Urol 1999;162:647-54. http://dx.doi.org/10.1097/00005392-199909010-00002

Correspondence: Dr. Alaya Yassein, Division of Urology, McMaster University, Hamilton, ON; ayassein@gmail.com 